\title{
VALUES FOR COOPERATIVE GAMES OVER GRAPHS AND GAMES WITH INADMISSIBLE COALITIONS
}

\author{
ZIV HELLMAN AND RON PERETZ
}

\begin{abstract}
We suppose that players in a cooperative game are located within a graph structure, such as a social network or supply route, that limits coalition formation to coalitions along connected subsets within the graph. This in turn leads to a more general study of coalitional games in which there are arbitrary limitations on the collections of coalitions that may be formed. Within this context we define a generalisation of the Shapley value that is studied from an axiomatic perspective. The resulting 'graph value' (and 'S-value' in the general case) is endogenously asymmetric, with the automorphism group of the graph playing a crucial role in determining the relative values of players.
\end{abstract}

Keywords: Shapley value, network games. JEL classification: C71, D46, D72.

\section{INTRODUCTION}

One of the standard interpretations of the Shapley value, as a measure of the average marginal contribution of a player to each and every possible coalition, may strain credulity if taken too literally in a great many social situations. This holds particularly when players may, due to affinity, consanguinity or other factors, have clear preferences for joining certain coalitions as opposed to others. Consider, for just one example, a job market. Is it not more likely that a potential hire will join a company if he knows someone within the company? How likely is it for a job seeker to join a company if she does not share a common language with any of its current employees?

Cases in which many theoretically possible coalitions will not realistically be formed are not limited to social situations alone. If one is studying cooperative coalitions amongst players connected via supply routes, computer networks or web links, there are clear structural reasons for entirely

Date: this version: April 16, 2015.

Department of Economics, Bar Ilan University; Department of Mathematics, London School of Economics email: ziv.hellman@biu.ac.il, ronprtz@gmail.com 
excluding some coalitions that would otherwise play a role in the calculation of the classic Shapley value and including in consideration instead only coalitions that are connected along the underlying network.

Networks, for obvious reasons, have increasingly been a focus of study in several disciplines over the past two decades. ${ }^{1}$ What we propose here is introducing network or graph structures directly into the study of coalitional game theory, by limiting consideration of potential coalitions solely to coalitions that are connected along the graph. Doing so, in the tradition of measuring average marginal contributions, yields different values that we argue may be more appropriate for assessing the values of players in many situations than the classic Shapley value.

The potential applications of a graph value are many. A partial list may include: coalition formation in complex political situations; studying power relations and cost sharing in situations with geographic constraints such as supply routes along roads or rivers; coalition formation in social networks; and perhaps even cooperation between neighbouring genes inside chromosomes.

This requires departing in some ways from the classic model of transferable utility games, which associates a certain worth to every coalition. That model implicitly assumes that the only force that drives the formation of coalitions is the worth they generate. The model we introduce here takes into account a proximity relation between players represented as edges of an undirected graph (a symmetric binary relation). It is assumed that a player only joins a coalition if he is connected to one of its members. As a result the only admissible coalitions are the connected subgraphs.

Once one has taken this step, however, it is natural to continue on to consider coalitional games in which some coalitions are inadmissible and cannot be formed, for arbitrary reasons, not necessarily because they are not connected along a path in a network. There may be many natural reasons for such a restriction. For example, there may be a cost incurred for the very formation of a coalition, depending on coalition membership, size and so forth. If the cost is too prohibitive, some coalitions may never be formed and should therefore be considered 'inadmissible'. Past history or cultural and social taboos may in some cases also place the formation of certain coalitions entirely out of the realm of possibility.

\footnotetext{
${ }^{1}$ Perhaps a contemporary canonical example would be an on-line social network, with coalitions naturally growing in size by way of adding at each stage friends of current members.
} 
This leads to the formal consideration of games with admissible structures, that is, games in which only a subset $\mathcal{A}$ of the set $2^{N}$ of all potential coalitions is admissible. ${ }^{2}$ In such games, the characteristic function is defined only for coalitions in $\mathcal{A}$, yielding a different theory from that of classical coalitional game theory.

Imposing the standard Shapley axioms (additivity, symmetry, null player and efficiency) to games with admissible structures, we define a value concept that we term an S-value. It might appear naïvely that doing so would easily yield a unique value generalising the Shapley value. Matters, however, turn out to be far from this simple. Even the existence of an S-value in a general game with an admissible structure is not always guaranteed; one must either assume further connectivity properties or assume more axioms.

Strengthening the additivity axiom in the list of the Shapley axioms to linearity is both necessary and sufficient for the existence of an S-value (Theorem 1). This alone does not yet grant uniqueness. We provide a characterisation of uniqueness of the S-value in Theorem 2.

After this study of values over games with admissible structures, which is interesting in its own right, we can apply the results to the special case of admissibility structures defined by graphs. We term monotonic S-values adapted to such admissibility structures graph values.

However, hopes that in this way a unique graph value can always, or even in most cases, be defined are dashed by Theorem 3, which shows that the graph value is unique only over two graphs: the complete graph (where it is the classic Shapley value) and the cycle. In all other graphs, the standard axioms used for the study of the Shapley value are insufficient to pick out a unique graph value.

The results here are, to the best of our knowledge, the first attempts to extend the Shapley value in general to situations of limited coalition admissibility and in particular to games with graphs defining relationships between players. They shed new light on the axiomatics underlying the classic Shapley value and also indicate that the theory of values in games with admissibility structures is likely to be a rich theory, requiring the addition of more axioms to replicate the straightforward existence and uniqueness that make the classic Shapley value such a compelling solution concept.

\section{From the Shapley Value to Graph Values}

\subsection{Motivation.}

\footnotetext{
${ }^{2}$ It should be noted that we do not depart from the classic assumption of complete information. The study of coalitional games of incomplete information is important in its own right. See for example Forges and Serrano (2011).
} 
As motivation, we begin with consideration of a well-known interpretation of the classic Shapley value. We suppose as background the standard model of a coalitional game, i.e., a set $N$ of players, of cardinality $n$, is given, along with a characteristic function $v$ which is a real-valued function $v: 2^{N} \rightarrow \mathbb{R}$ with the convention that $v(\emptyset)=0$.

The players are asked to enter the room in a random order $i_{1}, i_{2}, \ldots, i_{n}$. As each player $i_{j}$ enters, he or she joins the coalition $S=\left\{i_{1}, i_{2}, \ldots, i_{j-1}\right\}$ comprised of the players who have previously entered.

One could then possibly assign player $i_{j}$ the marginal value that he or she adds to $S$, i.e., $v\left(S \cup i_{j}\right)-v(S)$. That, however, would introduce a dependency on a particular random order. To avoid this, the celebrated Shapley value of a player (Shapley (1953a)) is defined to be the expected marginal contribution he or she adds to a coalition formed under such a random ordering, with respect to the uniform distribution over all orderings of $N$.

Underlying this story are several implicit assumptions, including:

(1) The assumption that all possible coalitions, e.g., all subsets of $N$, are admissible for consideration and may be constructed.

(2) The assumption that coalitions are constructed monotonically, that is, that new players are always added to existing coalitions but players in a coalition never leave.

(3) The assumption that players are always added one at a time to existing coalitions.

As noted in the introduction, however, there are many natural situations cases in which the assumption that all possible coalitions are admissible is untenable. In what follows we therefore limit the coalitions that are admissible and consider values in that more general case. It also turns out that this alone is not sufficient; the ways in which coalitions can be constructed, by adding or subtracting players, are also important for the study of values over games with admissibility structures.

\subsection{Preliminaries.}

A finite set of players $N$ of cardinality $n=|N|$ is assumed fixed throughout.

In a classic coalitional game theory model, one would proceed at this point to define a characteristic function assigning a worth to every possible subset of $N$. However, since we wish to generalise the classic Shapley value, we restrict the collection of coalitions which may be formed in our model. A collection $\mathcal{A} \subseteq 2^{N}$ of subsets of $N$ that includes the empty 
set $\emptyset$ and the grand coalition $N$ defines an admissibility structure, denoted $(N, \mathcal{A})$.

The elements of $\mathcal{A}$ intuitively play the role of the coalitions that may be formed, excluding the elements of $2^{N} \backslash \mathcal{A}$ from consideration. They will therefore formally be termed admissible coalitions, although we will often just call them coalitions for short.

Only admissible coalitions have a defined worth in the model, which affects the range of possible games. A coalitional game over an admissibility structure $(N, \mathcal{A})$ is given by a characteristic function $v$ which is a realvalued function $v: \mathcal{A} \rightarrow \mathbb{R}$ with the convention that $v(\emptyset)=0$. Denote the collection of all coalitional games over a fixed admissibility structure $\mathcal{A}$ by $\mathcal{K}(N, \mathcal{A})$, or simply by $\mathcal{K}$ for short when $N$ and $\mathcal{A}$ are fixed and understood.

In the special case in which the admissibility structure $\mathcal{A}=2^{N}$, the above definitions reduce to the standard definitions used in classic coalitional game theory.

Symmetry, as determined by automorphisms, will play a major role in the study of $\mathcal{K}$. We denote by $\operatorname{Aut}(N, \mathcal{A})$ the set of all permutations over $N$ that preserve $\mathcal{A}$, meaning bijective mappings $\pi: N \rightarrow N$ such that $\{i \in N: \pi(i) \in A\} \in \mathcal{A}$, for all $A \in \mathcal{A}$. With tolerable abuse of notation, given a permutation $\pi: N \rightarrow N$ we also consider $\pi$ to be a mapping $\pi: 2^{N} \rightarrow 2^{N}$ by defining $\pi\left(\left\{i_{1}, i_{2}, \ldots, i_{k}\right\}\right)=\left\{\pi\left(i_{1}\right), \pi\left(i_{2}\right), \ldots, \pi\left(i_{k}\right)\right\}$. We will also abuse notation by sometimes writing $i$ instead of the singleton set $\{i\}$ when no confusion is possible, for the sake of readability.

We say that player $i \in N$ is a null player in a game $v \in \mathcal{K}$, if $v(S)=$ $v(S \cup i)$, whenever $S, S \cup i \in \mathcal{A}$. An $\mathcal{A}$ preserving function $f: N \rightarrow N$ defines an operator on games, $f \circ v(S)=v\left(f^{-1}(S)\right)$. A game $v \in \mathcal{K}$ is called monotonic if $v(S) \leq v(T)$, for every $S, T \in \mathcal{A}$ such that $S \subset T$.

\subsection{Generalising the Shapley Value.}

Conceptually, a value for a coalitional game is a way of assigning a payoff to every player in the game. A value for player $i$ on $\mathcal{K}$ is therefore a function $\varphi_{i}: \mathcal{K} \rightarrow \mathbb{R}$. Given a value for each player, a (group) value on $\mathcal{K}, \varphi=$ $\left(\varphi_{1}, \varphi_{2}, \ldots, \varphi_{n}\right)$, concatenates the individual values into a payoff vector in $\mathbb{R}^{N}$ for each game.

The classic Shapley value was defined for $\mathcal{A}=2^{N}$ in Shapley (1953a) using four axioms. These four Shapley axioms form the basis for our generalisation of Shapley's value to more general admissibility structures.

Definition 1. We say that $\varphi: \mathcal{K}(N, \mathcal{A}) \rightarrow \mathbb{R}$ is an $S$-value if it satisfies the following axioms:

$$
\text { Additivity: } \varphi(v+u)=\varphi(v)+\varphi(u) \text {, for every } v, u \in \mathcal{K} .
$$


Symmetry: $\varphi_{\pi(i)}(\pi \circ v)=\varphi_{i}(v)$, for every $i \in N, v \in \mathcal{K}$, and $\pi \in \operatorname{Aut}(N, \mathcal{A})$.

Null player: $\varphi_{i}(v)=0$ whenever $i$ is a null player in $v$.

Efficiency: $\sum_{i \in N} \varphi_{i}(v)=v(N)$, for every $v \in \mathcal{K}$.

We say that $\varphi$ is monotonic if it satisfies

Monotonicity: $\varphi_{i}(v) \geq 0$, for every player $i$ and every monotonic game $v$.

We say that $\varphi$ is linear if it satisfies

Linearity: $\varphi(a v+u)=a \varphi(v)+\varphi(u)$, for every $v, u \in \mathcal{K}$ and $a \in \mathbb{R}$.

Note that additivity is a special case of linearity; the latter is a stronger assumption than the former.

The classic Shapley value is the special case of an S-value when $\mathcal{A}=$ $2^{N}$. In that case, Lloyd Shapley proved that existence and uniqueness are guaranteed. In the more general setting, existence might not hold, and it is natural to inquire under what conditions S-values exist and when they are unique.

As noted above, the proofs in Shapley (1953a) make use only of the properties of the additivity, symmetry, null player and efficiency axioms. The 'players entering a room one by one' explanation of the Shapley value as expected marginal contribution is a later interpretation. It makes implicit use of a 'connectivity' property inherent in the case $\mathcal{A}=2^{N}$, namely that adding one player to an already existing coalition always yields a new, admissible coalition.

As we will show, it turns out that in the general setting the existence of an S-value depends exactly on assuming a connectivity property for the admissibility structure. We say that $(N, \mathcal{A})$ is connected if there exists a finite sequence of admissible coalitions $\emptyset=S_{0}, S_{1}, \ldots, S_{k}=N$ such that $\left|\left(S_{l} \backslash S_{l-1}\right) \cup\left(S_{l-1} \backslash S_{l}\right)\right|=1$ for every $1 \leq l \leq k$. Such a sequence is called a chain.

The definition of a chain deliberately invokes the player-by-player construction of coalitions taken from the intuitive interpretation of the Shapley value. It is, however, more general in that the chain process admits the possibility of coalitions shrinking as well as expanding.

Given a chain $c=\left(S_{0}, S_{1}, \ldots, S_{k}\right)$ it is natural to define a value $\psi^{c}$ by

$$
\psi_{i}^{c}(v)=\sum_{l=1}^{k} \mathbf{1}\left(i \in\left(S_{l} \backslash S_{l-1}\right) \cup\left(S_{l-1} \backslash S_{l}\right)\right)\left[v\left(S_{l}\right)-v\left(S_{l-1}\right)\right],
$$


A (linear) S-value is then defined as

$$
\varphi^{c}=\sum_{\pi \in A u t(N, \mathcal{A})} \psi^{\pi(c)},
$$

where $\pi(c):=\left(\pi\left(S_{0}\right), \ldots, \pi\left(S_{k}\right)\right)$.

In general, $\varphi^{c}$ need not be monotonic, but if $S_{0} \subset S_{1} \subset \cdots \subset S_{k}$ (equivalently, $k=n$, i.e., the coalition construction process monotonically adds one new player at each step) then $\varphi^{c}$ is monotonic. Such a chain is called monotonic. If a monotonic chain for $(N, \mathcal{A})$ exists, we say that $(N, \mathcal{A})$ is monotonically connected.

It turns out that the existence of chains is necessary for the existence of the corresponding values. The following observations are further explained in Section 3.

Proposition 1. An admissibility structure admits an $S$-value if and only if it is connected.

Proposition 2. An admissibility structure admits a monotonic S-value if and only if it is monotonically connected.

Every solution concept that satisfies additivity and the null player property is linear over $\mathbb{Q}$. Monotonicity implies continuity; therefore any monotonic $\mathrm{S}$-value is linear.

The relation between (additive) $\mathrm{S}$-values and linear $\mathrm{S}$-values is as follows. Any $S$-value is linear over $\mathbb{Q}$. Let $\mathcal{K}_{1}$ be the linear space over $\mathbb{Q}$ of $\mathbb{Q}$-valued games. The space of real-valued games $\mathcal{K}$ can be represented as a direct sum of copies of $\mathcal{K}_{1}$. An $\mathrm{S}$-value on $\mathcal{K}$ is obtained by independently specifying it on each one of the copies of $\mathcal{K}_{1}$. Specifying the same value on each one of the copies yields a linear $\mathrm{S}$-value (on $\mathcal{K}$ ).

Consequently, both the existence and the uniqueness of an S-value coincide with the existence and the uniqueness of a linear $\mathrm{S}$-value. We can thus concentrate on the class of linear $S$-values in our quest for conditions on existence and uniqueness of $\mathrm{S}$-values in general. This is the content of Theorem 1.

Theorem 1. An admissibility structure admits an S-value if and only if it admits a linear $S$-value. Furthermore, an $S$-value is uniquely defined if and only if a linear $S$-value is uniquely defined.

In order to attain a similar relation between linear and monotonic Svalues, we assume an additional connectivity condition. We say that an admissibility structure $(N, \mathcal{A})$ is well-connected if there is a monotonic chain through any admissible coalition. In other words, for every admissible coalition $S$ there is a monotonic chain $S_{0} \subsetneq S_{1} \subsetneq \cdots \subsetneq S_{n}$, such that 
$S=S_{l}$ for some $0 \leq l \leq n$. On a well-connected admissibility structure, the set of all linear $\mathrm{S}$-values is the affine span of the set of all monotonic $\mathrm{S}$-values; therefore we have the following theorem:

Theorem 2. With respect to any well-connected admissibility structure, the following statements are equivalent:

(1) The $S$-value is uniquely defined.

(2) Every $S$-value is monotonic.

(3) Every $S$-value is linear.

(4) Every linear $S$-value is monotonic.

\subsection{Graph Values.}

Our original motivation for studying values over admissibility structures was consideration of coalition formation over graphs. Graphs provide a natural class of admissibility structures: a coalition over a graphs is admissible only if it can be constructed step-by-step by following a path in the graph. More formally, a connected graph $G=(N, E)$ whose vertex set is the set of players defines an admissibility structure $(N, \mathcal{A}(G))$, where $\mathcal{A}(G)$ is the collection of all connected subsets $S \subset N$. Namely, between any $i, j \in S$ there is a path of $G$ that visits only vertices of $S$. A monotonic S-value adapted to such an admissibility structure is called a graph value.

By Theorem 2, a graph $G$ admits a unique graph value if and only if it admits a unique $\mathrm{S}$-value. We immediately know at least one graph over $N$ that admits a unique graph value: the admissibility structure $\left(N, 2^{N}=\right.$ $\mathcal{A}\left(K_{n}\right)$ ) of the complete graph $K_{n}$ over $N$ (i.e., the graph in which each player is connected by an edge to every other player) is exactly the set $2^{N}$. The graph value in this case is therefore the unique classic Shapley value.

Which other graphs over $N$ admit a unique graph value? Surprisingly, apart from the complete graph there is only one other graph that admits a unique graph value.

Theorem 3. The graphs on which the graph value is uniquely defined are exactly the complete graph $K_{n}$ and the cycle $C_{n}$.

On the way to proving the above theorems we characterise the values that satisfy subsets of the axioms. These results are presented in Section 3 and summarised in Table 1.

\section{VAlues on Admissibility Structures}

\subsection{Coalitional DAG and n-linear values.}

An admissibility structure $(N, \mathcal{A})$ is associated with a directed acyclic graph (DAG) $D^{\mathcal{A}}=(\mathcal{A}, E(\mathcal{A}))$ whose vertex set is $\mathcal{A}$, and there is an arc 


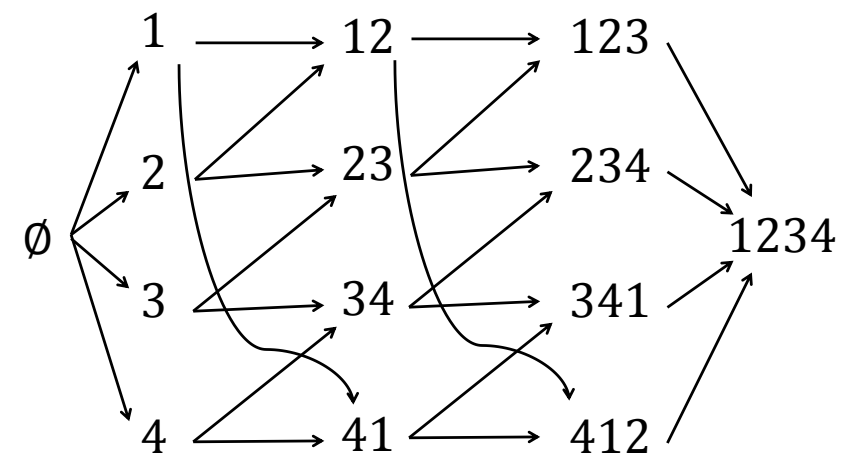

FIGURE 1. The directed acyclic graph associated with the 4-cycle. The admissible coalitions are subsets of $\mathbb{Z}_{4}$ of the form $\{i, i+1 \ldots, i+k\}, 0 \leq i, k \leq 3$.

from $S$ to $T$ if and only if $T=S \cup i$, for some $i \in N \backslash S$ (see an example in Figure 1). Note that an undirected path from $\emptyset$ to $N$ corresponds to a chain, a directed path - to a monotonic chain, and the fact that there are exactly one source and one sink - to $(N, \mathcal{A})$ being well-connected.

The values that satisfy the axioms of linearity and null player correspond to assignments of real numbers on the arcs of the coalitional DAG.

Before proving the above statement, let us fix some useful notation. Let $\left\{\chi_{T}\right\}_{T \in \mathcal{A} \backslash\{\emptyset\}}$ be the standard basis for $\mathcal{K}=\mathcal{K}(N, \mathcal{A})$ over $\mathbb{R}$. That is,

$$
\chi_{T}(S)= \begin{cases}1 & \text { if } S=T, \\ 0 & \text { if } S \neq T .\end{cases}
$$

For a collection of coalitions $\mathcal{B} \subset \mathcal{A} \backslash\{\emptyset\}$, we define $\chi_{\mathcal{B}}:=\sum_{T \in \mathcal{B}} \chi_{T}$, with tolerable abuse of notation. We also denote $\chi_{\supseteq T}:=\chi_{\{S \in \mathcal{A}: S \supseteq T\}}$ and $\chi_{\supsetneq T}:=\chi_{\{S \in \mathcal{A}: S \supsetneq T\}}$.

For a coalition $T \in \mathcal{A}$, let $T^{+}$be the set of all players $i \in N \backslash T$ such that $T \cup i \in \mathcal{A}$. Similarly, let $T^{-}$be the set of all players $i \in T$ such that $T \backslash i \in \mathcal{A}$. For $i \in N$, we define $A^{-i}=\left\{T \in \mathcal{A}: i \in T^{+}\right\}$.

If $\varphi: \mathcal{K} \rightarrow \mathbb{R}^{N}$ is linear, we have

$$
\varphi_{i}(v)=\varphi_{i}\left(\sum_{T \in \mathcal{A}} v(T) \chi_{T}\right)=\sum_{T \in \mathcal{A}} v(T) \varphi_{i}\left(\chi_{T}\right) .
$$


If $i \in T^{+}$, then $i$ is a null player in $\chi_{\{T, T \cup i\}}$; therefore if $\varphi$ satisfies linearity and the null player axiom, we have $\varphi_{i}\left(\chi_{T}\right)+\varphi_{i}\left(\chi_{T \cup i}\right)=0$. Since the players not in $T^{+} \cup T^{-}$are null players in $\chi_{T}$, Equation (2) becomes

$$
\varphi_{i}(v)=\sum_{T \in \mathcal{A}: i \in T^{+}} p_{T}^{i}(v(T \cup i)-v(T)),
$$

where $p_{T}^{i}=\varphi_{i}\left(\chi_{T \cup i}\right)$.

We call values that satisfy both the null-player and the linearity axioms n-linear values. From Equation (3) we get a one-to-one correspondence between the n-linear values on $(N, \mathcal{A})$ and functions from $E(\mathcal{A})$ to $\mathbb{R}$.

Proposition 3. The values satisfying the linearity and null-player axioms are exactly all the values of the form

$$
\varphi_{i}(v)=\sum_{T \in \mathcal{A}^{-i}} p_{T}^{i}[v(T \cup i)-v(T)]
$$

where $\left\{p_{T}^{i}: i \in N, T \in \mathcal{A}^{-i}\right\}$ are arbitrary real numbers.

In the rest of this section, we gradually introduce further axioms and examine the constraints that these axioms impose on the possible values of $\left\{p_{T}^{i}\right\}$.

\subsection{The dummy axiom and probabilistic values.}

The dummy axiom asserts that the value of a player whose marginal contribution is constant is exactly that constant. Formally, a player $i$ is called a dummy player with marginal contribution $d_{i} \in \mathbb{R}$ in a game $v \in \mathcal{K}(N, \mathcal{A})$, if $\mathcal{A}^{-i} \neq \emptyset$ ( $i$ has a marginal contribution) and $v(T \cup i)-v(T)=d_{i}$, for every $T \in \mathcal{A}^{-i}$.

If $d_{i}=0$ then $i$ is also a null player. If $d_{i} \neq 0$, we say that $i$ is a proper dummy player.

We say that $\varphi: \mathcal{K} \rightarrow \mathbb{R}^{N}$ satisfies the dummy axiom if

Dummy: $\varphi_{i}(v)=d_{i}$, whenever $i$ is a dummy player with marginal contribution $d_{i}$ in $v$.

From Equation (3) and the fact that whenever $\mathcal{A}^{-i} \neq \emptyset$ there exists a game in which $i$ is a proper dummy player (e.g., $\chi_{\{T \in \mathcal{A}: i \in T\}}$ ), we see that postulating the dummy axiom for n-linear values is equivalent to imposing the following constraint:

$$
\sum_{T \in \mathcal{A}^{-i}} p_{T}^{i}=1, \quad \text { for every player } i \text { for whom } \mathcal{A}^{-i} \neq \emptyset .
$$


Proposition 4. The values satisfying the linearity, null-player and dummy axioms are exactly all the values of the form

$$
\varphi_{i}(v)=\sum_{T \in \mathcal{A}^{-i}} p_{T}^{i}[v(T \cup i)-v(T)]
$$

where $\left\{p_{T}^{i}: i \in N, T \in \mathcal{A}^{-i}\right\}$ are real numbers satisfying

$$
\sum_{T \in \mathcal{A}^{-i}:} p_{T}^{i}=1
$$

for every $i \in N$ such that $A^{-i} \neq \emptyset$.

Following Weber (1988), a probabilistic value is a function $\varphi: \mathcal{K} \rightarrow \mathbb{R}^{N}$ given by Equation (3) that satisfies Equation (4) and

$$
p_{T}^{i} \geq 0, \quad \text { for every } i \text { and } T \in \mathcal{A}^{-i} .
$$

If we allow $p_{T}^{i}$ to be negative (imposing only Equation (4)), we say that $\varphi$ is a pre-probabilistic value.

Imposing Equation (5) is equivalent to postulating monotonicity for nlinear values.

Proposition 5. The monotonic values satisfying the linearity and null-player axioms are exactly all the values of the form

$$
\varphi_{i}(v)=\sum_{T \in \mathcal{A}^{-i}} p_{T}^{i}[v(T \cup i)-v(T)],
$$

where $\left\{p_{T}^{i}: i \in N, T \in \mathcal{A}^{-i}\right\}$ are non-negative real numbers.

Proof. If all $p_{T}^{i} \geq 0$ then Equation (3) yields a monotonic value. It remains to show the converse: every monotonic n-linear value $\varphi$ satisfies $\varphi_{i}\left(\chi_{T \cup i}\right) \geq 0$, for all $i \in N$ and $T \in \mathcal{A}^{-i}$. Take such $\varphi, i$, and $T$. Since $i$ is a null player in both $\chi_{\{T, T \cup i\}}$ and $\chi_{\supseteq T}$, we have $0=\varphi_{i}\left(\chi_{T}\right)+\varphi_{i}\left(\chi_{T \cup i}\right)=$ $\varphi_{i}\left(\chi_{T}\right)+\varphi_{i}\left(\chi_{\supsetneq T}\right)$, and so $\varphi_{i}\left(\chi_{T \cup i}\right)=\varphi_{i}\left(\chi_{\supsetneq T}\right)$. The game $\chi_{\supsetneq T}$ is monotonic; therefore $\varphi_{i}\left(\chi_{\supsetneq T}\right) \geq 0$.

Propositions 4 and 5 together yield the following corollary.

Corollary 1. A function $\varphi: \mathcal{K} \rightarrow \mathbb{R}^{N}$ is

- a pre-probabilistic value if and only if it satisfies the linearity, null player, and dummy axioms;

- a probabilistic value if and only if it satisfies the linearity, null player, dummy and monotonicity axioms. 


\subsection{Efficiency, flows, and random order values.}

We now introduce efficiency. First we remark that, in conjunction with n-linearity, efficiency implies the dummy axiom. To see that, suppose $\varphi$ is an efficient n-linear value. Let $v \in \mathcal{K}(N, \mathcal{A})$ be a game in which player $i$ is a dummy player with marginal contribution $d_{i}$. Let $u=d_{i} \chi_{\{T \in \mathcal{A}: i \in T\}}$. Player $i$ is a null player in $v-u$; therefore $\varphi_{i}(v)=\varphi_{i}(u)$. All of the players other than $i$ are null players in $u$, and so $\varphi_{i}(u)=u(N)=d_{i}$, by efficiency.

Efficient $\mathrm{n}$-linear values are related to flows on the coalitional DAG. Let $\varphi: \mathcal{K}(N, \mathcal{A}) \rightarrow \mathbb{R}^{N}$ be an n-linear value associated with weights $\left\{p_{T}^{i}:\right.$ $\left.i \in N, T \in \mathcal{A}^{-1}\right\}$ through Equation (3). If $\varphi$ is efficient, then for any $T \in \mathcal{A} \backslash\{\emptyset\}$ we have

$$
\chi_{T}(N)=\sum_{i \in N} \varphi_{i}\left(\chi_{T}\right)
$$

Since

$$
\chi_{T}(N)=\left\{\begin{array}{ll}
0 & \text { if } T \neq N, \\
1 & \text { if } T=N,
\end{array} \quad \varphi_{i}\left(\chi_{T}\right)= \begin{cases}p_{T \backslash i}^{i} & \text { if } i \in T^{-}, \\
-p_{T \cup i}^{i} & \text { if } i \in T^{+}, \\
0 & \text { otherwise }\end{cases}\right.
$$

we have

$$
\sum_{i \in T^{-}} p_{T}^{i}=\sum_{i \in T^{+}} p_{T \cup i}^{i}, \quad \text { for all } T \in \mathcal{A} \backslash\{\emptyset, N\},
$$

and

$$
\sum_{i \in N^{-}} p_{N}^{i}=1
$$

Conversely, if $\left\{p_{T}^{i}: i \in N, T \in \mathcal{A}^{-1}\right\}$ satisfies Equations (6) and (7), then the associated n-linear value is efficient. Indeed, for every $v \in$ $\mathcal{K}(N, \mathcal{A})$

$$
\begin{array}{r}
\sum_{i \in N} \varphi_{i}(v)=\sum_{i \in N} \sum_{T \in \mathcal{A}^{-i}} p_{T}^{i}(v(T \cup i)-v(T)) \quad \text { (by Equation (3)) } \\
=\sum_{T \in \mathcal{A} \backslash\{\emptyset\}} v(T)\left[\sum_{i \in T^{-}} p_{T \backslash i}^{i}-\sum_{j \in T^{+}} p_{T}^{j}\right] \\
=v(N) \quad \text { (by Equations (6) and (7)). }
\end{array}
$$

An assignment of real numbers to the arcs of the coalitional DAG $\left\{p_{T}^{i}\right.$ : $\left.i \in N, T \in \mathcal{A}^{-1}\right\}$ that satisfies Equation (6) is called a flow (from the source $\emptyset$ to the sink $N)$. A flow that satisfies Equation (7) is called a unit flow. If 
all the weights satisfy the constraint that $p_{T}^{i} \geq 0$, then the flow is called a non-negative (unit) flow.

Proposition 6. The values satisfying the linearity, null-player, and effciency axioms are exactly all the values of the form

$$
\varphi_{i}(v)=\sum_{T \in \mathcal{A}^{-i}} p_{T}^{i}[v(T \cup i)-v(T)]
$$

where $\left\{p_{T}^{i}: i \in N, T \in \mathcal{A}^{-i}\right\}$ constitutes a unit flow.

Corollary 2. The monotonic values satisfying the linearity, null-player, and efficiency axioms are exactly all the values of the form

$$
\varphi_{i}(v)=\sum_{T \in \mathcal{A}^{-i}} p_{T}^{i}[v(T \cup i)-v(T)],
$$

in which $\left\{p_{T}^{i}: i \in N, T \in \mathcal{A}^{-i}\right\}$ constitutes a non-negative unit flow.

Proof of Propostions 1 and 2. The proof follows from the fact that a (nonnegative) unit flow exists if and only if there is an un-directed (directed) path from $\emptyset$ to $N$ in $D^{\mathcal{A}}$.

Monotonic efficient $n$-linear values have the following interpretation: the players enter the room in a random order $i_{1}, i_{2}, \ldots, i_{n}$. The value of player $i$ is his or her expected marginal contribution with respect to that random order.

So far, this story follows the standard interpretation of the classic Shapley value. However, in our context, in order for the marginal contribution to be well defined, we must require that the players in the room at any given time form an admissible coalition (with probability one). This is equivalent to requiring that the coalitions $S_{k}=\left\{i_{1}, \ldots, i_{k}\right\}(k=0,1, \ldots, n)$ form a chain. Following Weber (1988), a value obtained in this way from a probability distribution over monotonic chains is called a random order value. Formally, for a monotonic chain $c$, the value $\psi^{c}$ (defined by Equation (1)) is a random order value; any convex combination of such values is called a random order value. An affine combination of random order values is called a pre-random order value.

Proposition 7. The monotonic values satisfying the linearity, null-player, and efficiency axioms are exactly the random order values.

Proof. For any monotonic chain $c, \psi^{c}$ satisfies linearity, null-player, efficiency, and monotonicity. Each one of these axioms is preserved under convex combinations; therefore random order values satisfy these axioms. 
Conversely, by Corollary 2, the set of monotonic efficient n-linear values correspond to the set of non-negative unit flows on the coalitional DAG. The set of non-negative unit flows is a convex polytope given by Equation (5), Equation (6), and Equation (7). The extreme points of this polytope are unit flows supported on a single directed path; therefore any non-negative unit flow is a convex combination of such paths. The proof is concluded with the observation that the value induced by any unit flow supported on a path $S_{0} S_{1} \cdots S_{n}$ is exactly $\psi^{S_{0}, S_{1}, \ldots, S_{n}}$.

Non-negative flows are supported on directed paths from the source $\emptyset$ to the sink $N$; therefore random order values do not depend on the worth of coalitions that are not contained in any monotonic chain. Removing these coalitions from $\mathcal{A}$ does not change the set of random order values. Let $(N, \mathcal{A})$ be a monotonically connected admissibility structure. Define $\mathcal{A}^{\prime} \subset \mathcal{A}$ as the largest sub-collection of coalition w.r.t. $\left(N, \mathcal{A}^{\prime}\right)$ being wellconnected. Explicitly,

$$
\mathcal{A}^{\prime}=\left\{T \in \mathcal{A}: D^{\mathcal{A}} \text { contains a directed path from } \emptyset \text { to } N \text { through } T\right\} .
$$

Proposition 8. The affine space of pre-random order values on a monotonically connected admissibility structure $(N, \mathcal{A})$ is isomorphic to the affine space of pre-random order values on $\left(N, \mathcal{A}^{\prime}\right)$ through the natural isomorphism

$$
\begin{aligned}
& \kappa:\left(\mathbb{R}^{N}\right)^{\mathcal{K}\left(N, \mathcal{A}^{\prime}\right)} \rightarrow\left(\mathbb{R}^{N}\right)^{\mathcal{K}(N, \mathcal{A})}, \\
& (\kappa \varphi)(v)=\varphi\left(v_{\mid \mathcal{A}^{\prime}}\right) .
\end{aligned}
$$

Any pre-random order value is efficient and n-linear. On well-connected admissibility structures, the converse is also true.

Proposition 9. The pre-random order values on a well-connected admissibility structure are exactly the efficient $n$-linear values.

Proof. The coalitional DAG of a well-connected admissibility structures has a single source $\emptyset$ and a single sink $N$. In light of Proposition 6 and Corollary 2, the proof follows from the combinatorial fact that on a finite directed graph with one source $s$ and one sink $t$, every unit flow from $s$ to $t$ is an affine combination of non-negative unit flows from $s$ to $t$ (Lemma 2).

From Propositions 8 and 9 we have the following corollary.

Corollary 3. The affine space of pre-random order values on a monotonically connected admissibility structure $(N, \mathcal{A})$ is isomorphic to the affine space of efficient $n$-linear values on $\left(N, \mathcal{A}^{\prime}\right)$ through the natural isomorphism $\kappa$ of Proposition 9. 
Lemma 2. Let $D$ be a finite directed graph with one source s and one sink $t$. Every unit flow from $s$ to $t$ is an affine combination of non-negative unit flows from $s$ to $t$.

Proof. Let $f: E(D) \rightarrow \mathbb{R}$ be a unit flow from $s$ to $t$. We prove the lemma by induction on the number of arcs $e$ such that $f(e)<0$. Let $e \in E(D)$ with $f(e)<0$. Extend the arc $e$ to a maximal simple directed path $p$. Since $s$ and $t$ are the only source and sink, $p$ is a simple directed path from $s$ to $t$. Let $g$ be the non-negative unit flow supported on $p$. We can express $f$ as an affine combination of unit flows

$$
f=(1+|f(e)|) \frac{f+|f(e)| g}{1+|f(e)|}-|f(e)| g,
$$

where $g$ is non-negative and $\frac{f+|f(e)| g}{1+|f(e)|}$ has one less negative edge than $f$.

3.4. The symmetry projection. Recall that $A u t(N, \mathcal{A})$ acts on games $v \in$ $\mathcal{K}(N, \mathcal{A})$ by

$$
(\pi \circ v)(T)=v\left(\pi^{-1}(T)\right) .
$$

By identifying vectors in $\mathbb{R}^{N}$ with additive games it is standard to define right and left actions of $\operatorname{Aut}(N, \mathcal{A})$ on values $\varphi: \mathcal{K}(N, \mathcal{A}) \rightarrow \mathbb{R}^{N}$ by

$$
\begin{aligned}
& (\pi \circ \varphi)(v)=\pi \circ(\varphi(v)), \\
& (\varphi \circ \pi)(v)=\varphi(\pi \circ v) .
\end{aligned}
$$

The conjugation action

$$
\varphi \mapsto \pi \circ \varphi \circ \pi^{-1}
$$

preserves each one of the axioms: additivity, linearity, null-player, dummy, efficiency, and monotonicity separately. Therefore, we can think of symmetry as the property of being a fixed point of a mixing operator Sym defined by

$$
\operatorname{Sym} \varphi=\frac{1}{|A u t(N, \mathcal{A})|} \sum_{\pi \in \operatorname{Aut}(N, \mathcal{A})} \pi \circ \varphi \circ \pi^{-1} .
$$

Note that a value is symmetric if and only if its conjugation orbit is a singleton.

The operator Sym is a projection from the set of values $\left(R^{N}\right)^{\mathcal{K}(N, A)}$ to the set of symmetric values $\operatorname{Sym}\left(\left(R^{N}\right)^{\mathcal{K}(N, A)}\right)$. Additionally, for any subset $X \subset\left(R^{N}\right)^{\mathcal{K}(N, A)}$ obtained by postulating a subset of the above axioms, $\operatorname{Sym}(X)$ is the set of symmetric values satisfying that subset of axioms.

Since Sym is a linear operator, we get the following characterisation of the linear S-values. 
Proposition 10. The monotonic $S$-values on a well-connected admissibility structure $(N, \mathcal{A})$ are exactly the random order values defined by $\operatorname{Aut}(N, \mathcal{A})$ invariant distributions over chains on $(N, \mathcal{A})$.

Proposition 11. With respect to a well-connected admissibility structure, the affine space of linear $S$-values is exactly the affine span of the set of monotonic $S$-values.

3.5. Additivity and Characterisation of S-values. In this section we finally present the proofs of Theorems 1 and 2 .

Proof of Theorem 1. Additivity and the null player axiom imply linearity over $\mathbb{Q}$. Denote the $\mathbb{Q}$-linear space of all rational games by

$$
\mathcal{K}_{1}:=\{v \in \mathcal{K}(N, \mathcal{A}): v(S) \in \mathbb{Q}, \forall S \in \mathcal{A}\} .
$$

For a real number $x \neq 0$ let

$$
\mathcal{K}_{x}:=x \mathcal{K}_{1}=\left\{v \in \mathcal{K}(N, \mathcal{A}): x^{-1} v(S) \in \mathbb{Q}, \forall S \in \mathcal{A}\right\} .
$$

For any $\mathbb{Q}$-linear function $\psi: \mathcal{K}(N, \mathcal{A}) \rightarrow \mathbb{R}^{N}$ and any $x \in \mathbb{R} \backslash\{0\}$, let $\psi_{x}$ be the restriction of $\psi$ to $\mathcal{K}_{x}$. Let $\varphi_{x}$ be the unique $\mathbb{R}$-linear extension of $\psi_{x}$ to $\mathcal{K}(N, \mathcal{A})$. Note that $\varphi_{x}$ is a linear over $\mathbb{R}$.

The transformation $\psi \mapsto \varphi_{x}$ preserves efficiency and symmetry, since linear extensions preserve properties defined by linear equalities (and symmetry is characterised as a fixed point of a linear operator).

We show that $\psi \mapsto \varphi_{x}$ preserves the null-player axiom. Suppose $i \in$ $N$ is a null player in a game $v=\sum_{T \in \mathcal{A}} r_{T} x \chi_{T}$. We can write $v=$ $\sum_{T \in \mathcal{A}^{-i}} r_{T} x \chi_{T, T \cup i}+\sum_{T: i \notin T^{-} \cup T^{+}} r_{T} x \chi_{T}$. Player $i$ is a null player in each one of the summands; therefore if $\psi$ satisfies the null player axiom, then so does $\varphi_{x}$, since

$$
\left(f_{x}\right)_{i}(v)=\sum_{T \in \mathcal{A}^{-i}} r_{T} \psi\left(x \chi_{T, T \cup i}\right)+\sum_{T: i \notin T^{-} \cup T^{+}} r_{T} \psi\left(x \chi_{T}\right)=0 .
$$

It follows that an admissibility structure admits an S-value iff it admits a linear S-value.

Suppose now that $\psi$ is an $\mathrm{S}$-value. Then $\varphi_{x}$, for each $x$, is a linear $\mathrm{S}$ value. If there is only one linear $\mathrm{S}$-value, then $\psi$ is uniquely defined on every $\mathcal{K}_{x}$, and since $\mathcal{K}(N, \mathcal{A})=\sum_{x \in \mathbb{R} \backslash\{0\}} \mathcal{K}_{x}, \psi$ is uniquely defined on $\mathcal{K}(N, \mathcal{A})$.

We next turn to the proof of Theorem 2 .

Proof of Theorem 2. The equivalence between the uniqueness of the Svalue and linear $\mathrm{S}$-value is established in Theorem 1. When they are not unique, one can take two different linear S-values $\varphi$ and $\psi$. Let $\mathcal{B} \subset \mathbb{R}$ 
be a basis for $\mathbb{R}$ over $\mathbb{Q}$. Take some $x \in \mathcal{B}$. Define a $\mathbb{Q}$-linear value $\eta$ by specifying, using a basis for $\mathcal{K}(N, \mathcal{A})$ over $\mathbb{Q}$,

$$
\eta\left(b \chi_{T}\right)= \begin{cases}\varphi\left(b \chi_{T}\right) & \text { if } b=x \\ \psi\left(b \chi_{T}\right) & \text { if } b \neq x .\end{cases}
$$

for every $b \in \mathcal{B}$ and $T \in \mathcal{A}$.

The restriction of $\eta$ to every $\mathcal{K}_{b}$ (for all $b \in \mathcal{B}$, using the denotation of Equation (9)) satisfies the Shapley axioms; therefore $\eta$ is an S-value. If $\eta$ were $\mathbb{R}$-linear, then we would have $\eta=\varphi$ since they agree on $\mathcal{K}_{x}$ which spans $\mathcal{K}(N, \mathcal{A})$ over $\mathbb{R}$. Similarly, we would have $\eta=\psi$, since they agree on some $\mathcal{K}_{b}, b \in \mathcal{B} \backslash\{x\}$. This would be a contradiction, hence $\eta$ is not $\mathbb{R}$-linear.

Well-connected admissibility structures always admit a monotonic value. By Proposition 5 the space of linear values is the affine span of the set of monotonic values. It follows that the uniqueness of the latter implies the uniqueness of the former. Since the set of monotonic S-values is bounded it can be equal to its affine span only if it is a single point.

\subsection{Summary of the axiomatics of values.}

Table 1 summarises the different classes of values obtained by postulating various subsets of the Shapley axioms on a well-connected admissibility structure.

\begin{tabular}{|l|l|}
\hline n-Linear Value +: & Equivalent to: \\
\hline dummy & pre-probabilistic value \\
\hline dummy, monotonicity & probabilistic value \\
\hline efficiency & pre-random order value \\
\hline efficiency, monotonicity & random order value \\
\hline efficiency, symmetry & $\operatorname{Aut}(N, \mathcal{A})$-invariant pre-r.o.v. \\
\hline efficiency, monotonicity, symmetry & $\operatorname{Aut}(N, \mathcal{A})$-invariant r.o.v. \\
\hline
\end{tabular}

TABLE 1. Axiomatics summary for well-connected admissibility structures.

\section{GRAPH VAlue}

Recall that a connected graph $G=(N, E)$ whose vertex set is the set of players defines an admissibility structure $(N, \mathcal{A}(G))$, where $\mathcal{A}(G)$ is the collection of all connected subsets of $N$. A graph value on $G$ is a monotonic S-value adapted to $(N, \mathcal{A}(G))$. 
Note that the automorphism group of the admissibility structure $\operatorname{Aut}(N, \mathcal{A}(G))$ is also the automorphism group of the graph $A u t(G)$. Any monotonic chain can be identified with an admissible ordering of the players, an enumeration of the players $\left\{i_{1}, i_{2}, \ldots, i_{n}\right\}=N$ such that $\left\{i_{1}, \ldots, i_{k}\right\}$ is connected for all $k=1, \ldots, n$. A probability distribution on the set of admissible orderings is called a random order, and it is called an invariant random order if it is invariant under the action of $A u t(G)$. By Proposition 10, the graph values on $G$ are exactly the invariant random order values, namely, random order values defined through invariant random orders.

Example 3 (Spectrum Value). Álvarez, Hellman and Winter (2013) introduced the spectrum value, a graph value defined over $P_{n}$, the path on $n$ vertices. Here, the edges are all pairs $\{k, k+1\}, k=1, \ldots, n-1$.

In this case the set of automorphisms contains only two elements: the identity mapping and the mapping that reverses the ordering of the players (so that player 1 is mapped to player $n$, player 2 to player $n-1$ and so on).

Example 4 (Classic Shapley Value). Let $K_{n}$ be the complete graph over $N$. Since the action of $\operatorname{Aut}\left(K_{n}\right)$ on the admissible orderings is transitive, there is only one invariant random order (the uniform distribution) in this case; therefore the graph value is uniquely defined.

This unique graph value in this case is precisely the classic Shapley value.

The proof of the uniqueness of the classic Shapley value in Example 4 relies on the fact that there is only one invariant random order over the complete graph. In fact, the complete graph is the only graph on which there is only one invariant random order. The graph value in general is not unique, mainly because there may be several invariant random orders.

Nevertheless there is one more graph, in addition to the complete graph, on which the graph value is uniquely defined - the cycle $C_{n}$. This holds true despite the fact that the cycle $C_{n}$ admits more than one invariant random order.

Example 5 ( $n$-cycle). An $n$-cycle, for $n \geq 3$ is the graph whose vertex set is $\{1, \ldots, n\}$ with edge set $E=\{\{1,2\},\{2,3\}, \ldots,\{n-1, n\},\{n, 1\}\}$.

Claim 1. The graph value over the $n$-cycle is unique for all $n \geq 3$.

Let $\varphi$ be any graph value over $C_{n}$. By construction, for each player $i$ there are exactly two players $j$ and $k$ that are connected to $i$ in $C_{n}$. Let $T \subset N$ be a connected coalition of players in $C_{n}$. Define $i$ to be an internal vertex of $T$ if each of the two players $j$ and $k$ connected to $i$ are also in $T$. 
Consider the unanimity game $\chi_{\supseteq T}$ with carrier $T \in \mathcal{A}\left(C_{n}\right) \backslash \emptyset$. If $i$ is an internal vertex of $T$ then $i$ is pivotal with respect to a given admissible ordering iff $i$ is the last player in that ordering. By symmetry, each internal player has an equal probability of being last; it follows that $\varphi_{i}\left(\chi_{\supseteq T}\right)=1 / n$ for all internal vertices $i$.

If $T$ has a boundary $(T \neq N)$, then the two players on the boundary are symmetric and they must therefore receive the same value by the symmetry axiom. The players outside $T$ are null player; therefore, by efficiency,

$$
\varphi_{j}\left(\chi_{\supseteq T}\right)=\frac{1}{2}\left(1-\frac{|T|-2}{n}\right)
$$

for each player $j$ on the boundary of $T$.

This is sufficient to show that the graph value is unique over the $n$-cycle, since the unanimity games span the space of all games. Note that the graph value on the $n$-cycle is different from the classic Shapley vale that assigns equal vales to all members of the carrier of a unanimity game.

Subsequently, in the proof of Theorem 3 we will show that the complete graph and the cycle are, in fact, the only graphs on which the graph value is unique.

Finally, we consider one more example of a graph with an interesting graph value.

Example 6 ( $n$-star). The $n$-star graph is defined over the vertex set $\{0,1,2, \ldots, n\}$ with edges $\{\{0,1\},\{0,2\}, \ldots,\{0, n\}\}$. Consider the simple majority game $v$ and any graph value $\psi$ over the $n$-star graph. Then straightforward combinatorial calculations show that

$$
\begin{aligned}
& \psi_{0}(v)=0 \\
& \psi_{1}(v)=\psi_{2}(v)=\cdots=\psi_{n}(v)=\frac{1}{n} .
\end{aligned}
$$

The result in Example 6 is again very different from the Shapley value, because the internal vertex receives a zero value under all circumstances. This is because the graph value essentially counts the number of times each player is a pivot player among all admissible orderings. In the simple majority game over the star graph, the internal node can never be the pivot player in any admissible coalition.

This may at first seem surprising, since one natural representation of the internal node of a star graph is a market maker through whom everyone else needs to go to conduct trade, or similarly a hub for resource distribution. One might think this would grant the internal player a great deal of power, 
yet the axioms that we assumed, which are almost verbatim adaptations of the standard Shapley axioms for our setting in which only connected coalitions may be formed, end up giving that player zero value.

One explanation for this phenomenon is as follows. In the standard Shapley value approach, measuring the average marginal gain a player causes by joining coalitions is entirely equivalent to measuring the average marginal loss he causes by leaving coalitions. In the graph value setting, this equivalence no longer obtains. Since only connected coalitions may be formed, leaving a coalition is only possible if the remaining coalition is connected.

\subsection{Proof of Theorem 3.}

The proof is based on a combinatorial lemma that uses the following ad hoc definition: we say that a connected graph $(N, E)$ satisfies the connencted complements $(C C)$ property, if for any connected subset of the vertices $A \subset N$, the complement $N \backslash A$ is connected, as well.

Lemma 7. The only graphs that satisfy the CC property are the complete graph $K_{n}$ and the cycle $C_{n}$.

Proof. Clearly the complete graph and the cycle satisfy CC. Let $G=$ $(N, E)$ be a connected graph satisfying CC. Let $\Delta$ be $G$ 's maximal degree. That is, $\Delta$ is the maximal number of neighbours of any single vertex in $N$.

If $\Delta \leq 2$, then $G$ is a collection of cycles, lines, and isolated vertices. Since $G$ is connected, it is either a cycle, a line, or a single vertex. Lines of more than three vertices do not satisfy $\mathrm{CC}$; therefore $G$ must be either a cycle, $K_{1}$, or $K_{2}$.

Assume now that $\Delta \geq 3$. The graph $G$ has a spanning tree $T$ with at least $\Delta$ vertices. One can find such a tree by starting at a maximal degree vertex, adding the edges around it, and completing to a spanning tree. Any two leaves of $T$ are connected by an edge of $G$, since the rest of the vertices are connected (through $T$ ).

Since the degree of any leaf is at most $\Delta$ and it is has edges to all other leaves plus one internal vertex, there are at most $\Delta$ leaves. It follows that there are exactly $\Delta$ leaves, and they are connected by vertex disjoint paths to the root vertex. If all of these paths are of length 1, i.e. $T$ is a star, then $G$ is a complete graph. Otherwise, at least one path is longer than 1. Say this path ends with the vertices $x^{\prime}$ followed by $x$. Let $y$ and $z$ be two other leaves of $T$. The tree $T^{\prime}=T-x^{\prime} x+y x$ is a spanning tree. Similarly to $T$, the leaves of $T^{\prime}$ are connected by edges of $G$. In particular, $z x^{\prime}$ is an edge of $G$, but this is a contradiction since $z$ cannot have more than $\Delta$ neighbours. 
Proof of Theorem 3 assuming Lemma 7. In one direction, the graph values on $K_{n}$ and $C_{n}$ are unique, since for $K_{n}$ the graph and the classic Shapley values coincide, and for $C_{n}$ the unique graph value is given by Claim 1.

The other direction of the proof proceeds in two steps.

Step 1. A graph $G$ is called a transitive if for every two vertices there is an automorphism of $G$ that maps one vertex to the other. The graph value is unique only on transitive graphs.

Supposing $G=(N, E)$ is a non-transitive graph, we show it has multiple values. Let $x, y \in N$ such that there is no automorphism of $G$ that maps $x$ to $y$. Let $c$ and $d$ be a chains that begins with $x$ and $y$ respectively. Let $\varphi$ be the random order value supported on $c$ 's orbit, and similarly $\psi$ the graph value supported on $d$ 's orbit. Consider the game $v$ in which the worth of any nonempty coalition is 1 . On one hand, $\varphi_{y}(v)=0$, since $y$ is never the first element of an automorphic image of $c$. On the other hand, $\psi_{y}(v)>0$, since $y$ is the first element of $d$. Therefore, $\varphi$ and $\psi$ are two different graph values.

Step 2. Any transitive graph that does not satisfy $\mathrm{CC}$ has multiple graph values.

Let $G=(N, E)$ be a connected transitive graph that does not satisfy CC. Let $A \subset N$ be a maximal connected set with respect to $N \backslash A$ being disconnected. If $N \backslash A$ contained more than two vertices, one of them could be added to $A$, and so $A$ would not be maximal. Therefore, $N \backslash A$ must consists of exactly two vertices, say $(x, y) \notin E$.

Let $c$ be an admissible ordering ending with $x$ and then $y$. Let $d$ be an admissible ordering ending with an edge. Let $\varphi$ be the random order value supported on $c$ 's orbit, and $\psi$ on $d$ 's orbit. Let $v$ be the unanimity game with carrier $A \cup\{x\}$. For $x$ to be pivotal in $v$ with respect to an admissible ordering, the ordering must end with either $x$ or $x y$. Since the graph is transitive the probability of the former event is $\frac{1}{|N|}$, under any invariant probability. Since $x y$ is not an edge, and $d$ ends with an edge, none of the orderings in $d$ 's orbit end with $x y$; therefore $\psi_{x}(v)=\frac{1}{|N|}$. The ordering $c$ does end with $x y$; therefore $\varphi_{x}(v)>\frac{1}{|N|}$, showing that $\varphi$ and $\psi$ are two different graph values.

From Steps 1 and 2, it follows that the graph value is unique only on graphs that satisfy CC. Lemma 7 says that these graphs are exactly $K_{n}$ and $C_{n}$. 


\section{REVIEW OF LITERATURE}

Our main inspiration, and the paper that is most similar in approach to this one, is Álvarez, Hellman and Winter (2013), which proposes a way to measure the relative power of political parties in a parliament by explicitly taking into account a political spectrum. That paper notes that it is highly unlikely for a left-wing party to form a coalition with a party holding strongly diametrical right-wing views unless there are other parties in the coalition that can 'bridge' the ideological differences. In more general terms, a political party will tend to join a pre-existing coalition only if the coalition contains at least one other party that is ideologically close to it. To formalise this idea, Álvarez, Hellman and Winter (2013) postulates that parties can be ordered along a political spectrum (i.e., a strict linear ordering), from right to left, and a coalition will form only if it consists of a consecutive range of ideological views along this spectrum. ${ }^{3}$

One possible shortcoming of that approach is that it may be artificial to ascribe all ideological differences to positioning along a single linear ordering. In practice, ideologies are often multidimensional, relating to several issues. That observation led to the model presented in this paper, which is a generalisation of the model in Álvarez, Hellman and Winter (2013). As an added benefit, by extending the underlying topology of the connections between players to any graph, the model here is potentially applicable to a very wide range of cooperative situations, including but by no means restricted to political-coalitional settings.

Weakening the axiom of symmetry for the sake of considering variations on the Shapley value is a very old idea. Weighted Shapley values were proposed by Lloyd Shapley himself in his seminal PhD thesis (Shapley (1953b)). Each weighted Shapley value associates a positive weight with each player. These weights are the proportions of the players' shares in unanimity games. The symmetric Shapley value is the special case in which all weights are the same. This concept was studied axiomatically in Kalai and Samet (1987).

The weights in these models, however, are imposed exogenously, representing some pre-existing measure of the relative strengths of the players which is then used for calculating weighted Shapley values. In contrast, in the approach here asymmetries arise endogenously from the positioning of the players along the underlying graph structure.

\footnotetext{
${ }^{3}$ As here, Álvarez, Hellman and Winter (2013) work with a weak version of symmetry and hence do not derive a unique value from the standard Shapley axioms alone. In that paper, an axiom reminiscent of various balanced contributions axioms, relating to unanimity games, needs to be added to attain uniqueness of the value.
} 
This paper is also far from the first to study situations in which not every coalition is feasible or equally likely. The issue is usually tackled by considering some structure on the set of players that circumscribes the way players can form coalitions. Games with these kind of structures are usually denoted games with restricted cooperation.

Among the earliest efforts in this direction, the beginnings of a large literature, are Aumann and Drèze (1975) and Owen (1977). These start from the supposition that cooperative games are endowed with a coalitional structure, an exogenously given partition of the players. When coalitions are formed, the players interact at two levels: first, bargaining takes place among the unions and then bargaining takes place inside each union. Within each union, however, every possible coalition is admissible.

Edelman (1997) and Bilbao and Edelman (2000) take an approach similar to the one adopted in the present paper, using geometric constraints to dictate which coalitions may be formed and which are deemed impossible. They, however, use the theory of convex geometries as the basis for their research, as opposed to the model of connected graphs used here. In respect to the axiomatics our treatment more closely resembles Shpaley's axiomatics as opposed to the more descriptive approach of Bilbao and Edelman (2000).

Graphs appear explicitly in Myerson (1977), but in a very different role from the one they have in this paper. There, an undirected graph describes communication possibilities between the players. A modification of the Shapley value is then proposed under the assumption that coalitions that are not connected in this graph are split into connected components. In that model too, within components all possible coalitions are admissible.

Myerson's model implicitly assumes superadditivity by granting (disconnected) coalitions the sums of the worths of their connected components. In our model disconnected coalitions are simply impossible, hence they do not assume any worth and we need not assume superadditivity.

Myerson (1977) assumes a fixed coalitional function while letting network structures vary, with axioms focussed on how allocation rules are related as the network structure changes. We consider the network as given, with our axioms focussed on how allocation rules are related as coalition functions vary, in the tradition of Shapley (1953a).

One may propose modelling impossible coalitions by setting their worths to zero while all other coalitions have positive worths. However, the choice of zero as the worth of impossible coalitions would be rather arbitrary and unjustified, as it makes the model variant under conditions of strategic equivalence. 
A situation in which the above is particularly problematic is cost sharing models (Megiddo, 1978; Granot and Huberman, 1981; Young, 1985). Consider, for example, organisations attempting to establish a communication network or supply route between themselves. Setting up a link between two organisations induces a cost. Due to physical constraints or geographic barrier, not every pair of organizations can be linked directly, while indirect connections via a sequence of links requires the active cooperation of all the organisations along these links. Examples may include a network of monetary transactions between banks or commodity flows between countries. The cost associated with a connected (admissible) coalition is the minimal total cost of links that connect the members of that coalition (minimal spanning tree). The cost of setting up the entire network has to be divided amongst the players (organisations).

The Shapley value, with its axioms interpreted as describing acceptable requirements for 'fair' cost allocation, has been proposed as the solution for cost sharing problems (the literature on this is vast, going back at least as far as Shubik (1962)). When some coalitions are deemed impossible, one could be tempted to compute an appropriate Shapley value by associating an extremely large cost with each impossible coalition. This approach does not work, since very large costs dictate heavy the costs in the resultant Shapley value. Some players will pay very large costs whereas others will receive very large payments (negative cost). As the costs associated with impossible coalitions grow, the actual costs of the links become negligible. The present paper proposes a solution that generalises the Shapley value to situations in which some coalitions are impossible while avoiding these potential conceptual pitfalls.

Jackson (2005) considers network games in which players can influence the structure of the network to serve their interests. Our model is different in that it exogenously imposes a fixed network structure.

\section{ACKNOWLEDGEMENTS}

We wish to thank Eyal Winter, Omer Edhan, and Yannai Gonczarowski for many helpful comments.

\section{REFERENCES}

Álvarez-Mozos, M., Z. Hellman and E. Winter (2013), Spectrum Value for Coalitional Games, Games and Economic Behavior, 82, pp. 132-142.

Aumann, R. J. and J. Drèze (1975), Cooperative Games with Coalition Structures, International Journal of Game Theory 4, pp. 217-237. 
VALUES FOR COOPERATIVE GAMES OVER GRAPHS AND GAMES WITH INADMISSIBLE COALITIORS

Bachrach, Y., Elkind, E., Meir, R., Pasechnik, D., Zuckerman, M., Rothe, J.; and Rosenschein, J. S. (2009), The Cost of Stability in Coalitional Games. In SAGT09, pp. 122-134.

Bilbao, J. M. (2000). Cooperative Games on Combinatorial Structures. Kluwer Publishers.

Bilbao, J. M. (2003), Cooperative Games Under Augmenting Systems, SIAM Journal on Discrete Mathematics 17(1), pp. 122-133.

Bilbao, J. M. and Edelman, P. H. (2000), The Shapley Value on Convex Geometries, Discrete Applied Mathematics 103, pp. 33-40.

Dubey, P. and R. J. Weber (1977), Probabilistic Values for Games, Cowles Foundation Discussion Paper 471, Yale University.

Edelman, P. H. (1997), A Note on Voting, Mathematics of Social Science 34, pp. 37-50.

Forges, F. and Serrano, R. (2011), Cooperative games with incomplete information: Some open problems, Working Paper 2011-14, Instituto Madrileño de Estudios Avanzados (IMDEA) Ciencias Sociales.

Granot, D. and Huberman, G. (1981), Minimum Cost Spanning Tree Games, Mathematical Programming 21 (1), pp. 1-18.

Jackson, M. O. (2005), Allocation rules for network games, Games and Economic Behavior 51 (1), pp. 128-154.

Kalai, E. and D. Samet (1997), On Weighted Shapley Values, International Journal of Game Theory 16 (3), pp. 205-222.

Megiddo, N. (1978), Computational complexity of the game theory approach to cost allocation on a tree, Mathematics of Operations Research 3 (3), pp. 189-196.

Myerson, R. B. (1977), Graphs and Cooperation in Games, Mathematics of Operations Research 2, pp. 673-692.

Owen, G. (1977), Values of Games with A-priori Unions, in Henn, R. and Moeschlin, O., editors, Essays in Mathematical Economics and Game Theory, pages 76588. Springer-Verlag.

Shapley, L. S. (1953), A Value for $N$-Person Games, in H. W. Kuhn and A. W. Tucker, editors, Contributions to the Theory of Games, Vol. II. Princeton University Press, Princeton, N.J.

Shapley, L. S. (1953), Additive and Non-Additive Set Functions, PhD thesis, Princeton University.

Shubik, M. (1962), Incentives, Decentralized Control, the Assignment of Joint Costs and Internal Pricing, Management Science 8, pp. 325-343.

Weber, R. J. (1988), Probabilistic Values for Games, in A. E. Roth, editor, The Shapley value: Essays in honor of Lloyd S. Shapley. Cambridge University Press, Cambridge, pp. 101119.

Young, P. (1985), Cost Allocation: Methods, Principles, Application. Amsterdam: North-Holland, 1985. 